\title{
A Delay fractioning Approach to Global Synchronization of Delayed Complex Networks with Stochastic Disturbances
}

\author{
Yao Wang $^{a}$, Zidong $\mathrm{Wang}^{a, b, *}$ and Jinling $\operatorname{Liang}^{c}$
}

\begin{abstract}
In this paper, the synchronization problem is investigated for a class of stochastic complex networks with time delays. By utilizing a new Lyapunov functional form based on the idea of 'delay fractioning', we employ the stochastic analysis techniques and the properties of Kronecker product to establish delay-dependent synchronization criteria that guarantee the globally asymptotically mean-square synchronization of the addressed delayed networks with stochastic disturbances. These sufficient conditions, which are formulated in terms of linear matrix inequalities (LMIs), can be solved efficiently by the LMI toolbox in Matlab. The main results are proved to be much less conservative and the conservatism could be reduced further as the number of delay fractioning gets bigger. A simulation example is exploited to demonstrate the advantage and applicability of the proposed result.
\end{abstract}

PACS: 05.45.Xt, 89.75.-k, 02.50.-r, 02.30.Ks.

\section{Keywords}

Global synchronization; stochastic complex networks; linear matrix inequality; Lyapunov functional; Kronecker product.

\section{INTRODUCTION}

Over the last few years, the complex networks have received increasing research attention from all fields of the basic science and the technological practice [1-10]. Complex networks can have applications in almost everywhere of the real world with examples including genetic networks, the Internet, and social networks etc. There have been a rich body of literature on analyzing complex networks, and one of the most significant dynamical behaviors of complex networks that has been widely investigated is the synchronization motion of its dynamical elements.

In practice, the information transmission within complex networks is in general not instantaneous since the signals traveling speed is limited. This fact gives rise to the time delays that may cause undesirable dynamic

This work was supported in part by the Engineering and Physical Sciences Research Council (EPSRC) of the U.K. under Grants GR/S27658/01, an International Joint Project sponsored by the Royal Society of the U.K., and the Alexander von Humboldt

Foundation of Germany.

${ }^{a}$ School of Information Sciences and Technology, Donghua University, Shanghai 200051, China.

${ }^{b}$ Department of Information Systems and Computing, Brunel University, Uxbridge, Middlesex, UB8 3PH, United Kingdom.

${ }^{c}$ Department of Mathematics, Southeast University, Nanjing 210096, China.

*Corresponding author. Email: Zidong.Wang@brunel.ac.uk, Fax: ++44/1895 251686. 
network behaviors such as oscillation and instability. It is worth pointing out that, among most existing results, the network synchronization problem has been predominantly studied for deterministic complex networks with or without delays, see $[2-5,9-14]$ and the references therein. For example, the global synchronization problem for complex networks without delays has been explored in $[2,4,5]$; the network synchronization problem of complex networks with delays or coupling delays has been studied in $[3,10,12,14]$; and the literature $[9,11,13]$ has been concerned with the adaptive synchronization problem of some dynamical networks.

In a real world, the signal transfer within complex networks could be perturbed randomly from the release of probabilistic causes such as neurotransmitters [15] and packet dropouts [16]. When analyzing the dynamical behaviors of complex networks, the obtained results are often largely affected by the stochastic disturbances. Subsequently, the synchronization problem for stochastic networks has begun to receive some initial research interests. In [17-21], the synchronization problems have been intensively investigated for delayed complex (or neural) networks with stochastic disturbances, where the criteria ensuring the synchronization among networks have been derived mainly based on the Lyapunov approach that is capable of coping the timedelays. Therefore, One of the main issues aroused here is how to reduce the possible conservatism induced by the introduction of the Lyapunov functional. Recently, the so-called 'delay fractioning' approach has been developed in $[22,23]$ that is shown to lead to much less conservative results than most existing literature. To the best of the authors' knowledge, the synchronization problem for stochastic delayed complex networks (SDCN) has not been fully investigated, and there still exists much room for further research such as the reduction of conservatism by using latest analysis techniques.

In this paper, we investigate the synchronization problem in an array of identical SDCN with time delays. By employing the properties of Kronecker product [24] and the stochastic analysis techniques [25, 26] combined with the 'delay fractioning' approach $[22,23]$, we construct a novel Lyapunov functional to attain new synchronization criteria, which are formulated in the form of linear matrix inequalities (LMIs) [27]. Note that the LMIs can be solved by using the standard numerical software. Our result is shown to be less conservative as the conservatism could be reduced when the number of delay fractions becomes bigger.

The remainder of this paper is organized as follows. In Section II, a stochastic complex network model with constant time delays is proposed and some preliminaries are briefly outlined. In Section III, by utilizing the approach of 'delay fractioning' and the Lyapunov functional method, we conduct the stochastic analysis to obtain delay-dependent sufficient criteria in terms of LMIs, so as to ensure the considered stochastic complex network with stochastic disturbances to be globally synchronized in the mean square. In Section IV, a simulation example is provided to show the advantage of the obtained result. The conclusions are finally drawn in Section V.

Notations: Throughout this paper, $\mathbb{R}^{n}$ and $\mathbb{R}^{n \times m}$ denote, respectively, the $n$ dimensional Euclidean space and the set of all $n \times m$ real matrices. $P>0$ means that matrix $P$ is real, symmetric and positive definite. $I$ and 0 denote the identity matrix and the zero matrix with compatible dimensions, respectively; and $\operatorname{diag}\{\cdots\}$ stands for a block-diagonal matrix. The superscript " $T$ " stands for matrix transposition and the asterisk "*" 
in a matrix is used to represent the term which is induced by symmetry. The Kronecker product of matrices $Q \in \mathbb{R}^{m \times n}$ and $R \in \mathbb{R}^{p \times q}$ is a matrix in $\mathbb{R}^{m p \times n q}$ and denoted as $Q \otimes R$. We let $\tau>0$ and $C\left([-\tau, 0] ; \mathbb{R}^{n}\right)$ denote the family of continuous functions $\varphi$ from $[-\tau, 0]$ to $\mathbb{R}^{n}$ with the norm $|\varphi|=\sup _{-\tau \leq \theta \leq 0}\|\varphi(\theta)\|$, where $\|\cdot\|$ is the Euclidean norm in $\mathbb{R}^{n}$. Moreover, let $\left(\Omega, \mathcal{F},\left\{\mathcal{F}_{t}\right\}_{t \geq 0}, P\right)$ be a complete probability space with a filtration $\left\{\mathcal{F}_{t}\right\}_{t \geq 0}$ satisfying the usual conditions (i.e., the filtration contains all $P$-null sets and is right continuous). Denote by $L_{\mathcal{F}_{0}}^{p}\left([-\tau, 0] ; \mathbb{R}^{n}\right)$ the family of all $\mathcal{F}_{0}$-measurable $C\left([-\tau, 0] ; \mathbb{R}^{n}\right)$-valued random variables $\xi=\{\xi(\theta):-\tau \leq \theta \leq 0\}$ such that $\sup _{-\tau \leq \theta \leq 0} \mathbb{E}|\xi(\theta)|^{p}<\infty$, where $\mathbb{E}\{\cdot\}$ stands for the mathematical expectation operator with respect to the given probability measure $P$. Sometimes, the arguments of a function will be omitted in the analysis when no confusion arises.

\section{PRoblem Formulation AND PRELiminaries}

Consider the following array of identical delayed complex networks with stochastic disturbances:

$$
\begin{aligned}
& d x_{i}(t)=\left[A x_{i}(t)+B f\left(x_{i}(t)\right)+B_{\tau} f\left(x_{i}(t-\tau)\right)+\sum_{j=1}^{N} G_{i j}^{(1)} \Gamma_{1} x_{j}(t)+\sum_{j=1}^{N} G_{i j}^{(2)} \Gamma_{2} x_{j}(t-\tau)\right] d t \\
& +\sigma_{i}\left(t, x_{i}(t), x_{i}(t-\tau)\right) d \omega(t), \quad i=1,2, \ldots, N,
\end{aligned}
$$

where $x_{i}(t)=\left(x_{i 1}(t), \ldots, x_{i n}(t)\right)^{T} \in \mathbb{R}^{n}$ is the state vector of the $i$ th network at time $t ; A$ denotes a known connection matrix; $B$ and $B_{\tau}$ denote, respectively, the connection weight matrix and the delayed connection weight matrix; $\Gamma_{1}, \Gamma_{2} \in \mathbb{R}^{n \times n}$ are matrices describing the inner-coupling between the subsystems at time $t$ and $t-\tau$, respectively; $G^{(1)}=\left(G_{i j}^{(1)}\right)_{N \times N}$ and $G^{(2)}=\left(G_{i j}^{(2)}\right)_{N \times N}$ are the outer-coupling configuration matrices representing the coupling strength and the topological structure of the complex networks. The constant $\tau$ stands for the constant time delay, which satisfies

$$
0 \leq \tau \leq h
$$

Furthermore, $\sigma_{i}(\cdot, \cdot, \cdot): \mathbb{R} \times \mathbb{R}^{n} \times \mathbb{R}^{n} \rightarrow \mathbb{R}^{n}$ is the noise intensity function vector, and $\omega(t)$ is a scalar Brownian motions defined on $(\Omega, \mathcal{F}, \mathcal{P})$ satisfying

$$
\mathbb{E}\{d \omega(t)\}=0 \text { and } \mathbb{E}\left\{[d \omega(t)]^{2}\right\}=d t
$$

Finally, $f\left(x_{i}(t)\right)=\left(f_{1}\left(x_{i 1}(t)\right), \ldots, f_{n}\left(x_{i n}(t)\right)\right)^{T}$ is an unknown but sector-bounded nonlinear function.

Throughout this paper, the following assumptions are needed.

Assumption 1: [3] The outer-coupling configuration matrices of the complex networks (1) satisfy

$$
G_{i j}^{(q)}=G_{j i}^{(q)} \geq 0 \quad(i \neq j), \quad G_{i i}^{(q)}=-\sum_{j=1, j \neq i}^{N} G_{i j}^{(q)} \quad(q=1,2 ; i, j=1,2, \ldots, N) .
$$

Assumption 2: $[15,28]$ For $\forall u, v \in \mathbb{R}^{n}$, the nonlinear function $f(\cdot)$ is assumed to satisfy the following sector-bounded condition

$$
\left(f(u)-f(v)-L_{f}(u-v)\right)^{T}\left(f(u)-f(v)-L^{f}(u-v)\right) \leq 0,
$$


where $L_{f}$ and $L^{f}$ are real constant matrices with $L^{f}-L_{f}$ being symmetric and positive definite.

Remark 1: The nonlinear function $f(\cdot)$ satisfying Assumption 2 is said to belong to the sector $\left[L_{f}, L^{f}\right]$ and it should be pointed out that this nonlinear condition is more general than the usually Lipschitz conditions which have been widely used in $[10,17,20]$.

Assumption 3: The noise intensity function vector $\sigma_{i}: \mathbb{R} \times \mathbb{R}^{n} \times \mathbb{R}^{n} \rightarrow \mathbb{R}^{n}$ satisfies the Lipschitz condition, i.e., there exist constant matrices $W_{1}$ and $W_{2}$ of appropriate dimensions such that the following inequality

$$
\left(\sigma_{i}\left(t, u_{1}, v_{1}\right)-\sigma_{j}\left(t, u_{2}, v_{2}\right)\right)^{T}\left(\sigma_{i}\left(t, u_{1}, v_{1}\right)-\sigma_{j}\left(t, u_{2}, v_{2}\right)\right) \leq\left\|W_{1}\left(u_{1}-u_{2}\right)\right\|^{2}+\left\|W_{2}\left(v_{1}-v_{2}\right)\right\|^{2}
$$

holds for all $i, j=1,2, \ldots, N$ and $u_{1}, v_{1}, u_{2}, v_{2} \in \mathbb{R}^{n}$.

Let

$$
\begin{aligned}
x(t) & =\left(x_{1}^{T}(t), x_{2}^{T}(t), \ldots, x_{N}^{T}(t)\right)^{T}, \\
F(x(t)) & =\left(f^{T}\left(x_{1}(t)\right), f^{T}\left(x_{2}(t)\right), \ldots, f^{T}\left(x_{N}(t)\right)\right)^{T}, \\
F(x(t-\tau)) & =\left(f^{T}\left(x_{1}(t-\tau)\right), f^{T}\left(x_{2}(t-\tau)\right), \ldots, f^{T}\left(x_{N}(t-\tau)\right)\right)^{T}, \\
\sigma(t) & =\left(\sigma_{1}^{T}\left(t, x_{1}(t), x_{1}(t-\tau)\right), \sigma_{2}^{T}\left(t, x_{2}(t), x_{2}(t-\tau)\right), \ldots, \sigma_{N}^{T}\left(t, x_{N}(t), x_{N}(t-\tau)\right)\right)^{T} .
\end{aligned}
$$

With the Kronecker product ' $\otimes$ ' for matrices, system (1) can be recast into

$$
\begin{gathered}
d x(t)=\left[\left(I_{N} \otimes A+G^{(1)} \otimes \Gamma_{1}\right) x(t)+\left(G^{(2)} \otimes \Gamma_{2}\right) x(t-\tau)+\left(I_{N} \otimes B\right) F(x(t))\right. \\
\left.+\left(I_{N} \otimes B_{\tau}\right) F(x(t-\tau))\right] d t+\sigma(t) d \omega(t) .
\end{gathered}
$$

The initial conditions associated with system (1) are given by

$$
x_{i}(s)=\varphi_{i}(s), \quad-h \leq s \leq 0, \quad i=1,2, \ldots, N
$$

where $\varphi_{i}(\cdot) \in L_{\mathcal{F}_{0}}^{2}\left([-h, 0], \mathbb{R}^{n}\right)$, and the corresponding state trajectory is denoted as $x_{i}\left(t, \varphi_{1}, \varphi_{2}, \ldots, \varphi_{N}\right)$.

Before stating the main results, some definitions and lemmas are introduced.

Definition 1: The set $\mathcal{S}=\left\{x=\left(x_{1}(s), x_{2}(s), \ldots, x_{N}(s)\right): x_{i}(s) \in L_{\mathcal{F}_{0}}^{2}\left([-h, 0], \mathbb{R}^{n}\right), x_{i}(s)=x_{j}(s), 1 \leq\right.$ $i, j \leq N\}$ is called the synchronization manifold of network (1) or (6).

Definition 2: The synchronization manifold $\mathcal{S}$ is said to be globally asymptotically stable in the mean square (in other words, the delayed complex network (1) is globally asymptotically synchronized in the mean square) if, for all $\varphi_{i}(\cdot), \varphi_{j}(\cdot) \in L_{\mathcal{F}_{0}}^{2}\left([-h, 0], \mathbb{R}^{n}\right)$, the following holds:

$$
\lim _{t \rightarrow \infty} \mathbb{E}\left\{\left\|x_{i}\left(t, \varphi_{i}\right)-x_{j}\left(t, \varphi_{j}\right)\right\|^{2}\right\}=0, \quad 1 \leq i<j \leq N .
$$

Lemma 1: [24] The Kronecker product has the following properties:

(1) $(\alpha A) \otimes B=A \otimes(\alpha B)$;

(2) $(A+B) \otimes C=A \otimes C+B \otimes C$;

$(3)(A \otimes B)(C \otimes D)=(A C) \otimes(B D)$;

(4) $(A \otimes B)^{T}=A^{T} \otimes B^{T}$. 
Lemma 2: [29] For scalar $r>0$, let $M \in \mathbb{R}^{m \times m}$ be a positive semi-definite matrix and $\rho:[0, r] \rightarrow \mathbb{R}^{m}$ be a vector function. If the integrations concerned are well defined, then the following inequality holds:

$$
r \int_{0}^{r} \rho^{T}(s) M \rho(s) d s \geq\left(\int_{0}^{r} \rho(s) d s\right)^{T} M\left(\int_{0}^{r} \rho(s) d s\right) .
$$

Lemma 3: Let $\mathcal{U}=\left(\alpha_{i j}\right)_{N \times N}, P \in \mathbb{R}^{n \times n}, x=\left(x_{1}^{T}, x_{2}^{T}, \ldots, x_{N}^{T}\right)^{T}$ where $x_{i}=\left(x_{i 1}, x_{i 2}, \ldots, x_{i n}\right)^{T} \in \mathbb{R}^{n}$ and $y=\left(y_{1}^{T}, y_{2}^{T}, \ldots, y_{N}^{T}\right)^{T}$ where $y_{i}=\left(y_{i 1}, y_{i 2}, \ldots, y_{i n}\right)^{T} \in \mathbb{R}^{n}(k=1,2, \ldots, N)$. If $\mathcal{U}=\mathcal{U}^{T}$ and each row sum of $\mathcal{U}$ is zero, then

$$
x^{T}(\mathcal{U} \otimes P) y=-\sum_{1 \leq i<j \leq N} \alpha_{i j}\left(x_{i}-x_{j}\right)^{T} P\left(y_{i}-y_{j}\right) .
$$

\section{MAin RESUlts AND PROOFS}

In this section, we are in the position to present our main results for synchronization criteria of the delayed complex networks with stochastic disturbances.

Theorem 1: Consider the complex network (1) with time-delay $\tau \in(0, h]$. For a given an integer $r \geq 1$, if there exist $2(r+1)$ matrices $P_{1}>0, P_{2}>0, Q_{k}>0, R_{k}>0, r+1$ matrices $M_{k}, S$, and $r+1$ positive scalars $\lambda, \epsilon_{k}(k=1,2, \ldots, r)$ such that the following LMIs hold for all $1 \leq i<j \leq N$ :

$$
\begin{aligned}
P_{1} & <\lambda I, \\
\Theta_{i j} & =W_{Q}^{T} \tilde{Q} W_{Q}+W_{R}^{T} \tilde{R} W_{R}+W_{S}^{T} \Lambda_{i j} W_{S}+W_{\varepsilon}^{T} \Xi W_{\varepsilon}<0,
\end{aligned}
$$

where

$$
\begin{aligned}
& W_{Q}=\left[\begin{array}{ccc}
I_{r n \times r n} & 0_{r n \times n} & 0_{r n \times(2 r n+2 n)} \\
0_{r n \times n} & I_{r n \times r n} & 0_{r n \times(2 r n+2 n)}
\end{array}\right], \quad W_{R}=\left[\begin{array}{ccc}
0_{r n \times(r n+n)} & I_{r n \times r n} & 0_{r n \times(r n+2 n)} \\
0_{r n \times(r n+2 n)} & I_{r n \times r n} & 0_{r n \times(r n+n)}
\end{array}\right], \\
& W_{\varepsilon}=\left[\begin{array}{ccc}
I_{r n \times r n} & 0_{r n \times(2 r n+3 n)} \\
0_{r n \times(r n+n)} & I_{r n \times r n} & 0_{r n \times(r n+2 n)} \\
0_{r n \times(2 r n+2 n)} & I_{r n \times r n} & 0_{r n \times n}
\end{array}\right], \quad W_{S}=\left[\begin{array}{ccc}
0_{n \times r n} & I_{n \times n} & 0_{n \times(2 r n+2 n)} \\
0_{n \times(r n+n)} & I_{n \times n} & 0_{n \times(2 r n+n)} \\
0_{n \times(2 r n+n)} & I_{n \times n} & 0_{n \times(r n+n)} \\
0_{n \times(3 r n+2 n)} & I_{n \times n}
\end{array}\right] \text {, } \\
& \tilde{Q}=\left[\begin{array}{cc}
\mathbf{Q}+\mathbf{M}+\mathbf{M}^{T} & -\mathbf{M} \\
* & -\mathbf{Q}
\end{array}\right], \quad \tilde{R}=\left[\begin{array}{cc}
\mathbf{R} & \\
& -\mathbf{R}
\end{array}\right], \quad \Xi=\left[\begin{array}{ccc}
-\varepsilon \otimes \hat{L} & \varepsilon \otimes \breve{L} & -\mathbf{M} \\
* & -2 \varepsilon \otimes I_{n} & 0 \\
* & * & -\frac{r}{h} \mathbf{P}_{\mathbf{2}}
\end{array}\right] \text {, }
\end{aligned}
$$




$$
\begin{aligned}
& \Lambda_{i j}=\left[\begin{array}{ccccc}
\Lambda_{i j}^{(11)} & -N G_{i j}^{(2)} P_{1} \Gamma_{2} & P_{1} B & P_{1} B_{\tau} & A^{T} S^{T}-N G_{i j}^{(1)} \Gamma_{1}^{T} S^{T} \\
* & \lambda W_{2}^{T} W_{2} & 0 & 0 & -N G_{i j}^{(2)} \Gamma_{2}^{T} S^{T} \\
* & * & 0 & 0 & B^{T} S^{T} \\
* & * & * & 0 & B_{\tau}^{T} S^{T} \\
* & * & * & * & h P_{2}-S-S^{T}
\end{array}\right], \\
& \Lambda_{i j}^{(11)}=P_{1} A+A^{T} P_{1}-N G_{i j}^{(1)}\left(P_{1} \Gamma_{1}+\Gamma_{1}^{T} P_{1}\right)+\lambda W_{1}^{T} W_{1}, \\
& \hat{L}=L_{f}^{T} L^{f}+L^{f^{T}} L_{f}, \breve{L}=\left(L_{f}^{T}+L^{f^{T}}\right), \mathbf{P}_{2}=I_{r} \otimes P_{2}, \varepsilon=\operatorname{diag}\left\{\epsilon_{1}, \epsilon_{2}, \ldots, \epsilon_{r}\right\}, \\
& \mathbf{Q}=\operatorname{diag}\left\{Q_{1}, Q_{2}, \ldots, Q_{r}\right\}, \mathbf{R}=\operatorname{diag}\left\{R_{1}, R_{2}, \ldots, R_{r}\right\}, \mathbf{M}=\operatorname{diag}\left\{M_{1}, M_{2}, \ldots, M_{r}\right\},
\end{aligned}
$$

then the asymptotic synchronization in the mean square in (8) is achieved.

Proof: By setting

$$
y(t)=\left(I_{N} \otimes A+G^{(1)} \otimes \Gamma_{1}\right) x(t)+\left(G^{(2)} \otimes \Gamma_{2}\right) x(t-\tau)+\left(I_{N} \otimes B\right) F(x(t))+\left(I_{N} \otimes B_{\tau}\right) F(x(t-\tau)),
$$

system (6) becomes

$$
d x(t)=y(t) d t+\sigma(t) d \omega(t)
$$

Based on the 'delay-fractioning' idea, we introduce the following new Lyapunov functional candidate for the complex network (1) (or (6)):

$$
V(t)=V_{1}(t)+V_{2}(t)+V_{3}(t)+V_{4}(t)
$$

where

$$
\begin{aligned}
V_{1}(t) & =x^{T}(t)\left(U \otimes P_{1}\right) x(t), \\
V_{2}(t) & =\int_{-\tau}^{0} \int_{t+\theta}^{t} y^{T}(s)\left(U \otimes P_{2}\right) y(s) d \theta d s, \\
V_{3}(t) & =\sum_{k=1}^{r} \int_{t-\frac{k}{r} \tau}^{t-\frac{k-1}{r} \tau} x^{T}(s)\left(U \otimes Q_{k}\right) x(s) d s, \\
V_{4}(t) & =\sum_{k=1}^{r} \int_{t-\frac{k}{r} \tau}^{t-\frac{k-1}{r} \tau} F^{T}(x(s))\left(U \otimes R_{k}\right) F(x(s)) d s,
\end{aligned}
$$

with $r \geq 1$ (number of fractions) being is an integer and

$$
U=\left[\begin{array}{cccc}
N-1 & -1 & \cdots & -1 \\
-1 & N-1 & \cdots & -1 \\
\cdots & \cdots & \cdots & \cdots \\
-1 & -1 & \cdots & N-1
\end{array}\right]_{N \times N}
$$


Letting $\mathscr{L}$ be the weak infinitesimal operator of the stochastic process $\left\{x_{t}=x(t+s) \mid t \geq 0,-h \leq s \leq 0\right\}$ along the trajectories of the network (1) (or (6)), then one has

$$
\begin{aligned}
& \mathscr{L} V_{1}(t)=2 x^{T}(t)\left(U \otimes P_{1}\right) y(t)+\sigma^{T}(t)\left(U \otimes P_{1}\right) \sigma(t) \\
& =2 x^{T}(t)\left(U \otimes P_{1}\right)\left[\left(I_{N} \otimes A+G^{(1)} \otimes \Gamma_{1}\right) x(t)+\left(G^{(2)} \otimes \Gamma_{2}\right) x(t-\tau)\right. \\
& \left.+\left(I_{N} \otimes B\right) F(x(t))+\left(I_{N} \otimes B_{\tau}\right) F(x(t-\tau))\right]+\sigma^{T}(t)\left(U \otimes P_{1}\right) \sigma(t) ; \\
& \mathscr{L} V_{2}(t)=\tau y^{T}(t)\left(U \otimes P_{2}\right) y(t)-\int_{t-\tau}^{t} y^{T}(s)\left(U \otimes P_{2}\right) y(s) d s \\
& \leq h y^{T}(t)\left(U \otimes P_{2}\right) y(t)-\int_{t-\tau}^{t} y^{T}(s)\left(U \otimes P_{2}\right) y(s) d s \\
& \mathscr{L} V_{3}(t)=x^{T}(t)\left(U \otimes Q_{1}\right) x(t)-x^{T}(t-\tau)\left(U \otimes Q_{r}\right) x(t-\tau) \\
& -\sum_{l=1}^{r-1}\left(x^{T}\left(t-\frac{l}{r} \tau\right)\left(U \otimes Q_{l}-U \otimes Q_{l+1}\right) x\left(t-\frac{l}{r} \tau\right)\right) \\
& =\left[\begin{array}{c}
x(t) \\
x\left(t-\frac{1}{r} \tau\right) \\
\vdots \\
x\left(t-\frac{r-1}{r} \tau\right)
\end{array}\right]^{T}\left[\begin{array}{llll}
U \otimes Q_{1} & & \\
& U \otimes Q_{2} & & \\
& & \cdots & \\
& & & U \otimes Q_{r}
\end{array}\right]\left[\begin{array}{c}
x(t) \\
x\left(t-\frac{1}{r} \tau\right) \\
\vdots \\
x\left(t-\frac{r-1}{r} \tau\right)
\end{array}\right] \\
& -\left[\begin{array}{c}
x\left(t-\frac{1}{r} \tau\right) \\
x\left(t-\frac{2}{r} \tau\right) \\
\vdots \\
x(t-\tau)
\end{array}\right]^{T}\left[\begin{array}{llll}
U \otimes Q_{1} & & & \\
& U \otimes Q_{2} & \\
& & \cdots & \\
& & & U \otimes Q_{r}
\end{array}\right]\left[\begin{array}{c}
x\left(t-\frac{1}{r} \tau\right) \\
x\left(t-\frac{2}{r} \tau\right) \\
\vdots \\
x(t-\tau)
\end{array}\right] \\
& \mathscr{L} V_{4}(t)=\left[\begin{array}{c}
F(x(t)) \\
F\left(x\left(t-\frac{1}{r} \tau\right)\right) \\
\vdots \\
F\left(x\left(t-\frac{r-1}{r} \tau\right)\right)
\end{array}\right]^{T}\left[\begin{array}{llll}
U \otimes R_{1} & & & \\
& U \otimes R_{2} & \\
& & \cdots & \\
& & & U \otimes R_{r}
\end{array}\right]\left[\begin{array}{c}
F(x(t)) \\
F\left(x\left(t-\frac{1}{r} \tau\right)\right) \\
\vdots \\
\\
\end{array}\right. \\
& -\left[\begin{array}{c}
F\left(x\left(t-\frac{1}{r} \tau\right)\right) \\
F\left(x\left(t-\frac{2}{r} \tau\right)\right) \\
\vdots \\
F(x(t-\tau))
\end{array}\right]^{T}\left[\begin{array}{llll}
U \otimes R_{1} & & & \\
& U \otimes R_{2} & & \\
& & \cdots & \\
& & & U \otimes R_{r}
\end{array}\right]\left[\begin{array}{c}
F\left(x\left(t-\frac{1}{r} \tau\right)\right) \\
F\left(x\left(t-\frac{2}{r} \tau\right)\right) \\
\vdots \\
F(x(t-\tau))
\end{array}\right] \text {. }
\end{aligned}
$$


From Lemma 2, it follows that

$$
\begin{aligned}
-\int_{t-\tau}^{t} y^{T}(s)\left(U \otimes P_{2}\right) y(s) d s & =-\sum_{k=1}^{r} \int_{t-\frac{k}{r} \tau}^{t-\frac{k-1}{r} \tau} y^{T}(s)\left(U \otimes P_{2}\right) y(s) d s \\
& \leq-\sum_{k=1}^{r}\left[\left(\frac{\tau}{r}\right)^{-1}\left(\int_{t-\frac{k}{r} \tau}^{t-\frac{k-1}{r} \tau} y(s) d s\right)^{T}\left(U \otimes P_{2}\right)\left(\int_{t-\frac{k}{r} \tau}^{t-\frac{k-1}{r} \tau} y(s) d s\right)\right] \\
& \leq-\frac{r}{h} \sum_{k=1}^{r}\left[\left(\int_{t-\frac{k}{r} \tau}^{t-\frac{k-1}{r} \tau} y(s) d s\right)^{T}\left(U \otimes P_{2}\right)\left(\int_{t-\frac{k}{r} \tau}^{t-\frac{k-1}{r} \tau} y(s) d s\right)\right] .
\end{aligned}
$$

From the Newton-Leibniz formula, we have that for any matrices $M_{k}(k=1,2, \ldots, r)$,

$$
2 x^{T}\left(t-\frac{k-1}{r} \tau\right)\left(U \otimes M_{k}\right)\left(x\left(t-\frac{k-1}{r} \tau\right)-x\left(t-\frac{k}{r} \tau\right)-\int_{t-\frac{k}{r} \tau}^{t-\frac{k-1}{r} \tau} y(s) d s-\int_{t-\frac{k}{r} \tau}^{t-\frac{k-1}{r} \tau} \sigma(s) d \omega(s)\right)=0 .
$$

In addition, for any matrix $S$, the following is true:

$$
\begin{aligned}
\Pi=2 y^{T}(t)(U \otimes S)\left[\left(I_{N} \otimes A\right.\right. & \left.+G^{(1)} \otimes \Gamma_{1}\right) x(t)+\left(G^{(2)} \otimes \Gamma_{2}\right) x(t-\tau) \\
& \left.+\left(I_{N} \otimes B\right) F(x(t))+\left(I_{N} \otimes B_{\tau}\right) F(x(t-\tau))-y(t)\right]=0 .
\end{aligned}
$$

Noting that $U G^{(i)}=G^{(i)} U=N G^{(i)}(i=1,2)$, for any matrix $H$ with appropriate dimension, we obtain

$$
(U \otimes H)\left(G^{(i)} \otimes \Gamma_{i}\right)=\left(U G^{(i)}\right) \otimes\left(H \Gamma_{i}\right)=\left(N G^{(i)}\right) \otimes\left(H \Gamma_{i}\right) .
$$

Combining (13)-(19) together with the property (20), we have

$$
\begin{aligned}
& \mathscr{L} V(t) \\
= & \sum_{1 \leq i<j \leq N}\left[\left(x_{i}(t)-x_{j}(t)\right)^{T}\left(P_{1} A+A^{T} P_{1}-N G_{i j}^{(1)}\left(P_{1} \Gamma_{1}+\Gamma_{1}^{T} P_{1}\right)\right)\left(x_{i}(t)-x_{j}(t)\right)\right. \\
& +2\left(x_{i}(t)-x_{j}(t)\right)^{T}\left(P_{1} B\left(f\left(x_{i}(t)\right)-f\left(x_{j}(t)\right)\right)+P_{1} B_{\tau}\left(f\left(x_{i}(t-\tau)\right)-f\left(x_{j}(t-\tau)\right)\right)\right. \\
& \left.-N G_{i j}^{(2)} P_{1} \Gamma_{2}\left(x_{i}(t-\tau)-x_{j}(t-\tau)\right)\right)+\left(y_{i}(t)-y_{j}(t)\right)^{T}\left(h P_{2}-S-S^{T}\right)\left(y_{i}(t)-y_{j}(t)\right) \\
& +\left(\sigma_{i}\left(t, x_{i}(t), x_{i}(t-\tau)\right)-\sigma_{j}\left(t, x_{j}(t), x_{j}(t-\tau)\right)\right)^{T} P_{1}\left(\sigma_{i}\left(t, x_{i}(t), x_{i}(t-\tau)\right)-\sigma_{j}\left(t, x_{j}(t), x_{j}(t-\tau)\right)\right) \\
& -2\left(\Upsilon_{i}(t)-\Upsilon_{j}(t)\right)^{T} \mathbf{M}\left(\left(\Upsilon_{i}\left(t-\frac{1}{r} \tau\right)-\Upsilon_{j}\left(t-\frac{1}{r} \tau\right)\right)+\left(\mathscr{P}_{i}(t)-\mathscr{P}_{j}(t)\right)+\left(\Omega_{i}(t)-\Omega_{j}(t)\right)\right) \\
& -\frac{r}{h}\left(\mathscr{P}_{i}(t)-\mathscr{P}_{j}(t)\right)^{T} \mathbf{P}_{\mathbf{2}}\left(\mathscr{P}_{i}(t)-\mathscr{P}_{j}(t)\right)+2\left(\left(x_{i}(t)\right)-x_{j}(t)\right)^{T}\left(A^{T} S^{T}-N G_{i j}^{(1)} \Gamma_{1}^{T} S^{T}\right)\left(y_{i}(t)-y_{j}(t)\right) \\
& -2\left(\left(x_{i}(t-\tau)\right)-x_{j}(t-\tau)\right)^{T} N G_{i j}^{(2)} \Gamma_{2}^{T} S^{T}\left(y_{i}(t)-y_{j}(t)\right)+2\left(f\left(x_{i}(t)\right)-f\left(x_{j}(t)\right)\right)^{T} B^{T} S^{T}\left(y_{i}(t)-y_{j}(t)\right) \\
+ & 2\left(f\left(x_{i}(t-\tau)\right)-f\left(x_{j}(t-\tau)\right)\right)^{T} B_{\tau}^{T} S^{T}\left(y_{i}(t)-y_{j}(t)\right)+\left(\Upsilon_{i}(t)-\Upsilon_{j}(t)\right)^{T}\left(\mathbf{Q}+\mathbf{M}+\mathbf{M}^{T}\right)\left(\Upsilon_{i}(t)-\Upsilon_{j}(t)\right) \\
& -\left(\Upsilon_{i}\left(t-\frac{1}{r} \tau\right)-\Upsilon_{j}\left(t-\frac{1}{r} \tau\right)\right)^{T} \mathbf{Q}\left(\Upsilon_{i}\left(t-\frac{1}{r} \tau\right)-\Upsilon_{j}\left(t-\frac{1}{r} \tau\right)\right)+\left(\mathscr{F}_{i}(t)-\mathscr{F}_{j}(t)\right)^{T} \mathbf{R}_{(}\left(\mathscr{F}_{i}(t)-\mathscr{F}_{j}(t)\right) \\
& -\left(\mathscr{F}_{i}\left(t-\frac{1}{r} \tau\right)-\mathscr{F}_{j}\left(t-\frac{1}{r} \tau\right)^{T} \mathbf{R}\left(\mathscr{F}_{i}\left(t-\frac{1}{r} \tau\right)-\mathscr{F}_{j}\left(t-\frac{1}{r} \tau\right)\right)\right],
\end{aligned}
$$


where $\mathbf{P}_{\mathbf{2}}, \mathbf{Q}, \mathbf{R}, \mathbf{M}$ are defined in (10), and

$$
\begin{aligned}
\Upsilon_{i}(t)= & {\left[\begin{array}{c}
x_{i}(t) \\
x_{i}\left(t-\frac{1}{r} \tau\right) \\
\vdots \\
x_{i}\left(t-\frac{r-1}{r} \tau\right)
\end{array}\right], \mathscr{F}_{i}(t)=\left[\begin{array}{c}
f\left(x_{i}(t)\right) \\
f\left(x_{i}\left(t-\frac{1}{r} \tau\right)\right) \\
\vdots \\
f\left(x_{i}\left(t-\frac{r-1}{r} \tau\right)\right)
\end{array}\right], \mathscr{P}_{i}(t)=\left[\begin{array}{c}
\int_{t-\frac{1}{r} \tau}^{t} y_{i}(s) d s \\
\int_{t-\frac{2}{r} \tau}^{t-\frac{\tau}{r}} y_{i}(s) d s \\
\vdots \\
\int_{t-\tau}^{t-\frac{r-1}{r} \tau} y_{i}(s) d s
\end{array}\right], } \\
\Omega_{i}(t)= & {\left[\begin{array}{c}
\int_{t-\frac{1}{r} \tau}^{t} \sigma(s) d \omega(s) \\
\int_{t-\frac{1}{r} \tau}^{t-\frac{1}{r} \tau} \sigma(s) d \omega(s) \\
\vdots \\
\int_{t-\tau}^{t-\frac{r-1}{r} \tau} \sigma(s) d \omega(s)
\end{array}\right], }
\end{aligned}
$$

According to (5) and (9), it is clear that

$$
\begin{aligned}
& \left(\sigma_{i}\left(t, x_{i}(t), x_{i}(t-\tau)\right)-\sigma_{j}\left(t, x_{j}(t), x_{j}(t-\tau)\right)\right)^{T} P_{1}\left(\sigma_{i}\left(t, x_{i}(t), x_{i}(t-\tau)\right)-\sigma_{j}\left(t, x_{j}(t), x_{j}(t-\tau)\right)\right) \\
& \leq \lambda\left[\left(x_{i}(t)-x_{j}(t)\right)^{T} W_{1}^{T} W_{1}\left(x_{i}(t)-x_{j}(t)\right)+\left(x_{i}(t-\tau)-x_{j}(t-\tau)\right)^{T} W_{2}^{T} W_{2}\left(x_{i}(t-\tau)-x_{j}(t-\tau)\right)\right] .
\end{aligned}
$$

Moreover, from Assumption 2, for $\epsilon_{k}>0(k=1,2, \cdots, r)$, it can be derived that

$$
\epsilon_{k}\left[\begin{array}{c}
x_{i}\left(t-\frac{k-1}{r} \tau\right)-x_{j}\left(t-\frac{k-1}{r} \tau\right) \\
f\left(x_{i}\left(t-\frac{k-1}{r} \tau\right)\right)-f\left(x_{j}\left(t-\frac{k-1}{r} \tau\right)\right.
\end{array}\right]^{T}\left[\begin{array}{cc}
\hat{L} & -\breve{L} \\
* & 2 I
\end{array}\right]\left[\begin{array}{c}
x_{i}\left(t-\frac{k-1}{r} \tau\right)-x_{j}\left(t-\frac{k-1}{r} \tau\right) \\
f\left(x_{i}\left(t-\frac{k-1}{r} \tau\right)-f\left(x_{j}\left(t-\frac{k-1}{r} \tau\right)\right.\right.
\end{array}\right] \leq 0,
$$

with $\hat{L}=L_{f}^{T} L^{f}+L^{f^{T}} L_{f}, \breve{L}=\left(L_{f}^{T}+L^{f^{T}}\right)$. Obviously, one has from (23) that

$$
\begin{aligned}
\left(\Upsilon_{i}(t)-\Upsilon_{j}(t)\right)^{T}(2(\varepsilon \otimes \breve{L}) & \left.\left(\mathscr{F}_{i}(t)-\mathscr{F}_{j}(t)\right)-(\varepsilon \otimes) \hat{L}\left(\Upsilon_{i}(t)-\Upsilon_{j}(t)\right)\right) \\
& -\left(\mathscr{F}_{i}(t)-\mathscr{F}_{j}(t)\right)^{T}\left(2 \varepsilon \otimes I_{n}\right)\left(\mathscr{F}_{i}(t)-\mathscr{F}_{j}(t)\right) \geq 0
\end{aligned}
$$

with $\varepsilon=\operatorname{diag}\left\{\epsilon_{1}, \epsilon_{2}, \ldots, \epsilon_{r}\right\}>0$.

Using (22) and (24), we obtain

$$
\mathbb{E}\{\mathscr{L} V(t)\} \leq \sum_{1 \leq i<j \leq N} \xi_{i j}^{T}(t) \Theta_{i j} \xi_{i j}(t)
$$

where

$$
\xi_{i j}(t)=\left[\begin{array}{c}
\Upsilon_{i}(t)-\Upsilon_{j}(t) \\
x_{i}(t-\tau)-x_{j}(t-\tau) \\
\mathscr{F}_{i}(t)-\mathscr{F}_{j}(t) \\
f\left(x_{i}(t-\tau)\right)-f\left(x_{j}(t-\tau)\right) \\
\mathscr{P}_{i}(t)-\mathscr{P}_{j}(t) \\
y_{i}(t)-y_{j}(t)
\end{array}\right]
$$

and $\Theta_{i j}$ is defined in (10). 
From (10), it is guaranteed that all the subsystems in (1) are asymptotically synchronized for any fixed time delay $\tau \in(0, h]$. The proof is completed.

Remark 2: Based on the 'delay fractioning' method, we construct a more general Lyapunov functional to analyze the synchronization problem of the delayed complex networks. The new delay-dependent conditions presented in Theorem 1 are formulated in the form of LMIs, which can be solved by the LMI toolbox in Matlab. Moreover, the conservatism of the results in Theorem 1 can be reduced by increasing the number of fractions of the time delay.

\section{A Numerical EXAmple}

In this section, we present a simulation example so as to illustrate the advantage and usefulness of our main results.

Example 1: Consider a coupled complex network (26) consisting of three identical models. The state equations of the entire array are

$$
\begin{aligned}
d x_{i}(t)= & {\left[A x_{i}(t)+B f\left(x_{i}(t)\right)+B_{\tau} f\left(x_{i}(t-\tau)\right)+\sum_{j=1}^{3} G_{i j}^{(1)} \Gamma_{1} x_{j}(t)+\sum_{j=1}^{3} G_{i j}^{(2)} \Gamma_{2} x_{j}(t-\tau)\right] d t } \\
& +\sigma\left(t, x_{i}(t), x_{i}(t-\tau) d \omega(t)\right.
\end{aligned}
$$

where $x_{i}(t)=\left(x_{i 1}(t), x_{i 2}(t)\right)^{T}(i=1,2,3)$ is the state vector of the $i$ th subsystem. Choose the coupling matrices $G^{(1)}, G^{(2)}$ and the linking matrices $\Gamma_{1}, \Gamma_{2}$ as

$$
G^{(1)}=\left[\begin{array}{ccc}
-2 & 1 & 1 \\
1 & -2 & 1 \\
1 & 1 & -2
\end{array}\right], G^{(2)}=\left[\begin{array}{ccc}
-3 & 1 & 2 \\
1 & -2 & 1 \\
2 & 1 & -3
\end{array}\right] ; \Gamma_{1}=\left[\begin{array}{cc}
0.5 & 0 \\
0.1 & 0.5
\end{array}\right], \Gamma_{2}=\left[\begin{array}{cc}
0.5 & 0.1 \\
0 & 0.4
\end{array}\right] \text {. }
$$

The other parameters are as follows:

$$
A=\left[\begin{array}{cc}
-2 & 0.2 \\
0.2 & -1.6
\end{array}\right], \quad B=\left[\begin{array}{cc}
0.6 & -0.1 \\
-0.3 & 0.5
\end{array}\right], \quad B_{\tau}=\left[\begin{array}{cc}
-0.5 & -0.1 \\
0.2 & -1.5
\end{array}\right] .
$$

The nonlinear function is given by $f(y(t))=\left(f_{1}\left(y_{1}(t)\right), f_{2}\left(y_{2}(t)\right)\right)^{T}$ with $f_{i}\left(y_{i}\right)=\tanh \left(y_{i}\right)(i=1,2)$ and the noise intensity function vector $\sigma(\cdot, \cdot, \cdot)$ is of the following form:

$$
\sigma(t, y(t), y(t-\tau))=\left[\begin{array}{cccc}
-0.05 & 0.05 & 0.1 & -0.1 \\
0.05 & -0.05 & 0.1 & -0.1
\end{array}\right]\left[\begin{array}{c}
y(t) \\
y(t-\tau)
\end{array}\right]
$$

Obviously, the nonlinear functions satisfy Assumptions 2-3 with

$$
L_{f}=\left[\begin{array}{ll}
0 & 0 \\
0 & 0
\end{array}\right], \quad L^{f}=\left[\begin{array}{ll}
1 & 0 \\
0 & 1
\end{array}\right] ; \quad W_{1}=\left[\begin{array}{cc}
0.2 & -0.2 \\
0.2 & -0.2
\end{array}\right], \quad W_{2}=\left[\begin{array}{cc}
-0.1 & 0.1 \\
0.1 & -0.1
\end{array}\right] .
$$

According to Theorem 1, the array of coupled delayed complex networks (1) with stochastic disturbances can achieve globally asymptotically synchronization in the mean square under the allowable maximum delay. 
By using the Matlab LMI Toolbox, LMIs (9)-(10) are feasible (the solutions are not given there for the purpose of space saving). When we set $r=1$, the time delay bound is 0.9475 , and when we take $r=2$, the allowable maximum delay increases to 0.959 . It can be seen that, the more the number of fractions, the less conservative the criterion is.

By randomly choosing the initial states in $[0,1] \times[0,1]$ and under the case $\tau=0.959$, the synchronization errors are plotted in Fig. 1 and Fig. 2, which confirm that the complex dynamical system (1) is globally synchronized in the mean square.

\section{Conclusions}

In this paper, we have dealt with the analysis problem for network synchronization of a class of stochastic delayed complex networks with $N$ identical subsystems. By employing a 'delay fractioning' approach, we have constructed a novel Lyapunov functional, and then we have used the properties of Kronecker product and stochastic analysis theory to obtain the synchronization conditions. The criteria derived in this paper are dependent on the allowable maximum delay, and it has been proved that the more the delay fractions are, the less conservative the result will be. Moreover, the LMI-based criteria can be verified by the standard numerical software. In the end of the paper, we have given an example to show the advantage and usefulness of our results.

\section{REFERENCES}

[1] C. Dangalchev, Physica A 338 (2004) 659.

[2] Z. Duan, G. Chen, L. Huang, Physics Letters A (in press, doi.org/10.1016/j.physleta.2008.02.056.)

[3] H. Gao, J. Lam, G. Chen, Physics Letters A 360 (2006) 263.

[4] Z. Li, G. Chen, IEEE Trans. Circuits Syst.-II 53 (2006) 28.

[5] W. Lu, T.Chen, Physica D 198 (2004) 148.

[6] S. Strogatz, Nature 410 (2001) 268.

[7] Z. Toroczkai, Los Alamos Science 29 (2005) 94.

[8] X. Wang, G. Chen, IEEE Circuits Syst. Mag. 3 (2003) 6.

[9] J. J. Yan, W. D Chang, M. L. Hung, Chaos, Solitons and Fractals 29 (2006) 506.

[10] W. Yu, J. Cao, J. Lu, SIAM J. Applied Dynamical Systems 7 (2008) 108.

[11] C. Hua, Q. Wang, X. Guan, Physics Letters A 368 (2007) 281.

[12] C.P. Li, W.G. Sun, J. Kurths, Physica A 361 (2006) 24.

[13] Z. Li, J. Lee, Physica A (in press, doi.10.1016/j.physa.2007.10.063.)

[14] X. Liu, T. Chen, Physica A 381 (2007) 82-92.

[15] Z. Wang, Y. Liu, M. Li, X. Liu, IEEE Trans. Neural Networks 17 (2006) 814.

[16] H. Gao, T. Chen, IEEE Trans. Auotmatic Control 53 (2008) 655.

[17] J. Liang, Z. Wang, X. Liu, Nonlinear Dynamics, 53 (2008) 153.

[18] J. Liang, Z. Wang, Y. Liu, X. Liu, IEEE Trans. Systems, Man, and Cybernetics - Part B (in press).

[19] Y. Sun, J. Cao, Z. Wang, Neurocomputing, 70 (2007) 2477.

[20] W. Wang, J. Cao, Physica A 366 (2006) 197.

[21] Z. Wang, H. Shu, J. Fang, X. Liu, Nonlinear Analysis: Real World Applications 7 (2006) 1119.

[22] S. Mou, H. Gao, W. Qiang, K. Chen, IEEE Trans. Systems, Man, and Cybernetics - Part B, 38 (2008) 571. 
[23] S. Mou, Y. Zhao, H. Gao, W. Qiang, Int. J. Computer Math. (in press).

[24] A. N. Langville, W. J. Stewart, Journal of Computational and Applied Mathematics 167 (2004) 429.

[25] L. Arnold, Random Dynamical Systems. Springer-Verlag, Berlin, 1998.

[26] R.Z. Khasminskii, Stochastic Stability of Differential Equations. Alphen aan den Rijn, Sijthoffand Noor, Khasminskiidhoff, 1980 .

[27] S. Boyd, L. E. Ghaoui, E. Feron and V. Balakrishnan, Linear Matrix Inequalities in System and Control Theory. Philadelphia: SIAM, 1994.

[28] Y. Liu, Z. Wang, X. Liu, Neural Networks 19 (2006) 667.

[29] K.Q. Gu, V.L. Kharitonov, J. Chen, Stability of time-delay systems. Boston: Birkhauser, 2003. 


\section{Captions:}

Figure 1: Synchronization error of $x_{i 1}(t)-x_{11}(i=2,3)$

Figure 2: Synchronization error of $x_{i 2}(t)-x_{12}(i=2,3)$ 


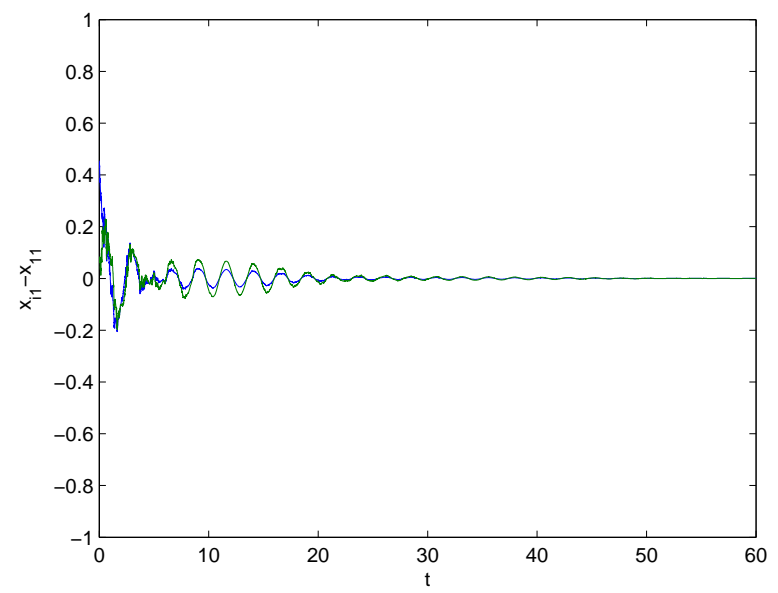

Fig. 1. Synchronization error of $x_{i 1}(t)-x_{11}(i=2,3)$

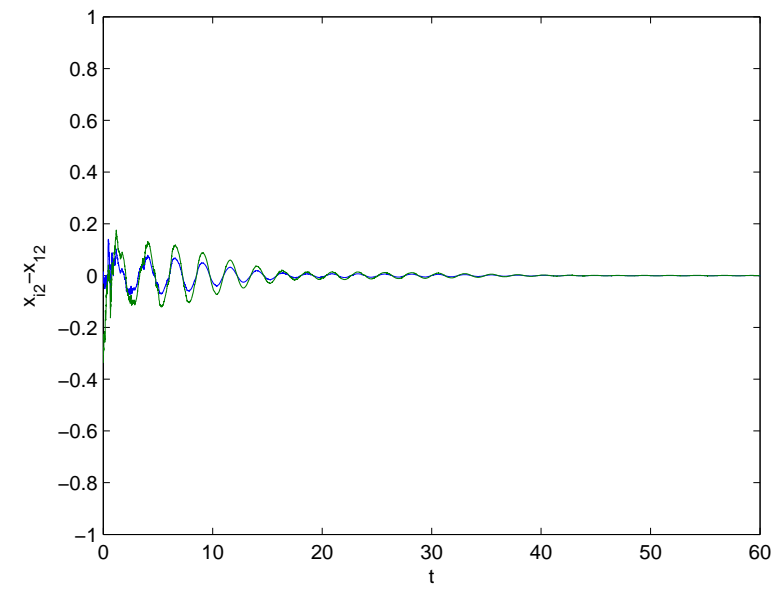

Fig. 2. Synchronization error of $x_{i 2}(t)-x_{12}(i=2,3)$ 